\title{
Pratiques politiques subalternes dans un quartier populaire de Tokyo: des formes de résistance?
}

Subaltern Political Practices in a Working Class Neighborhood of Tokyo: Forms of Resistance?

Nicolas Pinet

\section{OpenEdition}

\section{Journals}

Édition électronique

URL : http://journals.openedition.org/conflits/19145

DOI : 10.4000/conflits. 19145

ISSN : $1777-5345$

Éditeur :

CCLS - Centre d'études sur les conflits lilberté et sécurité, L'Harmattan

Édition imprimée

Date de publication : 19 mai 2016

Pagination : $35-56$

ISBN : 978-2-343-09459-5

ISSN : 1157-996X

\section{Référence électronique}

Nicolas Pinet, «Pratiques politiques subalternes dans un quartier populaire de Tokyo: des formes de résistance? », Cultures \& Conflits [En ligne], 101 | printemps 2016, mis en ligne le 19 mai 2017, consulté le 30 mars 2021. URL : http://journals.openedition.org/conflits/19145 ; DOI : https://doi.org/10.4000/ conflits. 19145 


\section{Pratiques politiques subalternes dans un quartier populaire de Tokyo : des formes de résistance?}

\section{Nicolas PINET}

Nicolas Pinet est chercheur postdoctoral au sein du département d'anthropologie de l'Université de Tokyo et membre du Laboratoire de changement social et politique de l'Université Paris Diderot - Paris 7. Ses recherches actuelles portent sur les pratiques politiques des classes populaires urbaines, dans une perspective comparative. Il a publié notamment Figures de la révolte : Rébellions latino-américaines (Syllepse, 2016, coordination) et "La Politique des profanes : Formes d'action politique et pratiques de citoyenneté des jeunes adultes " (Revue du MAUSS, $n^{\circ} 43$, 2014, pp. 395-409).

$\mathrm{D}$ omination et résistance sont devenues depuis une trentaine d'années des thèmes centraux dans le champ des sciences sociales ${ }^{1}$. Si la plupart des études prend pour objet privilégié l'un ou l'autre versant du couple ${ }^{2}$, certaines analyses adoptent aussi une perspective plus relationnelle en s'intéressant à ce que les pratiques de résistance nous apprennent sur les formes de pouvoir auxquelles elles se confrontent ${ }^{3}$. Pour le seul concept de résistance, qui nous intéresse plus particulièrement ici, les champs d'application sont très divers : résistances paysannes et résistances à l'État dans les travaux de James Scott ${ }^{4}$ et des

1. Je remercie les relecteurs et relectrices, connu-e-s et anonymes, de ce texte pour leur lecture attentive et leurs commentaires et suggestions.

2. La domination est par exemple un thème majeur des travaux de Pierre Bourdieu alors que la résistance est au centre de ceux de James Scott (voir notamment, pour ne citer que quelques travaux faisant des éléments du couple leur objet explicite : Bourdieu P., « Les modes de domination ", Actes de la recherche en sciences sociales, n²-3, 1976, pp. 122-132; Bourdieu P., La Domination masculine suivi de Quelques questions sur le mouvement gay et lesbien, Paris, Seuil, 2002 ; Scott J.C., Weapons of the Weak: Everyday Forms of Peasant Resistance, New Haven, Yale University Press, 1985 ; Scott J.C., Domination and the Arts of Resistance: Hidden Transcripts, New Haven, Yale University Press, 1990. Pour les œuvres en anglais, je cite directement, en traduisant si nécessaire, les versions originales, même si une traduction existe dans certains cas.

3. Abu-Lughod L., «The Romance of Resistance: Tracing Transformations of Power Through Bedouin Women ", American Ethnologist, vol. 17, n¹, février 1990, pp. 41-55.

4. Voir encore Scott J.C., The Art of Not Being Governed: An Anarchist History of Upland 
études rurales, résistances au travail, à la domination au travail, dans ceux des sociologues du travail 5, "droit de résistance à l'oppression », révoltes et révolutions dans les travaux de certains historiens et historiennes ${ }^{6}$... Puisque la résistance est conçue comme l'envers de la domination, les études dont c'est l'objet sont en général centrées sur les groupes et les individus situés du « mauvais côté » du rapport de pouvoir. Les études qui s'intéressent aux résistances des groupes et individus situés du «bon côté » du rapport de pouvoir mais dont les positions sont menacées sont nettement moins fréquentes 7 : l'imaginaire principal, en sciences sociales comme ailleurs, fait bien de la résistance l'autre - double antagoniste - de la domination. La retombée de la "vague contestataire ${ }^{8}$ » des années 1960 et 1970 semble avoir conduit, dans l'univers académique, à une réorientation parallèle des thématiques de recherche : la focalisation sur les formes massives et visibles de résistance émeutes paysannes, révoltes et révolutions - a été en partie relayée par un intérêt nouveau pour des formes moins spectaculaires, moins visibles et plus quotidiennes de résistance ${ }^{9}$. Le changement de focale dans les travaux de James Scott entre 1976 et 198510 témoigne de cette évolution. On dispose ainsi désormais d'un panorama assez complet des formes de luttes, ordinaires et extraordinaires, menées par les groupes subalternes ${ }^{11}$. Il en est de même dans le champ de la sociologie et de la géographie urbaines, avec des études focalisées tantôt sur les formes de résistance au quotidien ${ }^{12}$, tantôt sur des formes plus extra-ordinaires de résistance - révoltes, émeutes, mobilisations de masse ${ }^{13} \ldots$

Southeast Asia, New Haven, Yale University Press, 2009.

5. Bouquin S. (dir.), Résistances au travail, Paris, Syllepse, 2008 ; Bouquin S., «La question des résistances au travail dans la sociologie du travail française ", Actuel Marx, $\mathrm{n}^{\circ}$ 49, mai 2011, pp. 60-72 ; Calderón Gil J.A., Cohen V. (dir.), Qu'est-ce que résister ? : usages et enjeux d'une catégorie d'analyse sociologique, Villeneuve-d'Ascq, Presses universitaires du Septentrion, 2014.

6. Zancarini J.-C. (dir.), Le Droit de résistance : XII $I^{e}-X X^{e}$ siècle, Lyon, ENS éd., 1999 ; Gros D., Camy O. (dir.), «Le droit de résistance à l’oppression », Le Genre humain, n ${ }^{\circ} 4$, Paris, Seuil, 2005.

7. Voir notamment Viennot E., La France, les femmes et le pouvoir. II, Les résistances de la société (XVII'-XVIII siècle), Paris, Perrin, 2008.

8. Mathieu L., Les Années 70, un âge d'or des luttes? Paris, Textuel, 2009.

9. J'emprunte l'idée à Lila Abu-Lughod («The Romance of Resistance», op. cit., p. 41).

10. The Moral Economy of the Peasant: Rebellion and Subsistence in Southeast Asia, New Haven, Yale University Press, 1976 ; Weapons of the Weak, op. cit.

11. Le mot «subalterne» est pris ici en son sens étymologique - le sub-alternus est celui qui se trouve "sous " un autre, c'est-à-dire dans une position de subordination au sein de tel ou tel rapport de pouvoir. La métaphore spatiale des positions relatives qui donne son sens au mot est la même que celle que l'on trouve dans le couple domination et résistance.

12. Voir par exemple : Bayat A., Life as Politics: How Ordinary People Change the Middle East, Stanford, Stanford University Press, 2010 [2008] ; Giroud M., " "Résister en habitant" : les luttes dans les quartiers populaires à l'épreuve du renouvellement urbain ", Contretemps, $\mathrm{n}^{\circ} 13,2005$, pp. 49-58 et, pour une analyse plus approfondie, Giroud M., « Résister en habitant ? : renouvellement urbain et continuités populaires en centre ancien (Berriat Saint-Bruno à Grenoble et Alcântara à Lisbonne) », thèse de doctorat de géographie, Poitiers, 2007.

13. Castells M., The City and the Grassroots: A Cross-Cultural Theory of Urban Social Movements, Berkeley, University of California Press, 1983 ; Abu-Lughod J.L., Race, Space, and Riots in Chicago, New York, and Los Angeles, New York, Oxford University Press, [2007] 2012 ; Pinet N. (dir.), Figures de la révolte : Rébellions latino-américaines, XVIe-XXe siècles, Paris, Syllepse, 2016. 
Malgré sa fortune dans le champ des sciences sociales, la notion de résistance continue à poser un certain nombre de problèmes, notamment en ce qui concerne sa délimitation : jusqu'où faut-il considérer que telles ou telles pratiques constituent une "résistance » ? Le schéma conceptuel mobilisé restreint déjà l'extension de la notion puisque, comme indiqué plus haut, la résistance n'est le plus souvent étudiée que du côté des groupes subalternes, comme résistance à une forme ou une autre de pouvoir. Mais cela ne règle en rien l'épineuse question de l'intentionnalité. Faut-il considérer, avec Brian Fegan ${ }^{14}$, que sans intention de résister, il n'y a pas de résistance ? Une telle option risque cependant d'imposer un cadre conceptuel trop rigide sur des pratiques par nature en constante évolution : ce qui n'est pas d'abord considéré comme résistance par les acteurs ou les observatrices peut fort bien être perçu comme tel plus tard.

Cet article propose un positionnement possible dans le débat autour de l'intentionnalité sur la base d'une analyse de matériaux collectés dans le cadre d'une ethnographie (2012-2016) portant sur les pratiques politiques dans un quartier populaire de Tokyo. Pour les raisons explicitées au paragraphe suivant, ce texte se focalise sur un groupe social bien particulier : les personnes résidant dans des parcs ou dans différents espaces publics ou semi-publics de la zone. Pour faire référence à elles, je propose d'utiliser, par souci de précision, le substantif qu'elles emploient volontiers elles-mêmes : nojukusha (野宿者), qui désigne les individus qui «campent », «dorment à l'extérieur ». En effet, les termes de sans-abri ou de sans domicile fixe ne paraissent pas vraiment appropriés puisque, de fait, ils dorment bien dans des abris, plus ou moins solides et spacieux, construits souvent avec une armature de bois et des bâches bleues, et que, pour une partie significative d'entre eux, leur lieu de résidence est fixe...

Le caractère fondamentalement instable de leurs conditions d'existence qui reposent sur l'occupation d'espaces hors des dispositifs légaux habituels d'adjudication de l'espace - achat, location -, leur mode de vie se déployant, pour une part importante, en plein air et au grand jour, et la précarité de leurs situations rendent plus évident le repérage de pratiques politiques qui, si elles sont aussi présentes dans le cas des résidents des immeubles d'habitat social, sont à la fois moins visibles et moins fréquentes.

14. Fegan B., « Tenants' non-violent resistance to landowner claims in a central Luzon », The Journal of Peasant Studies, vol. 13, n² 2, janvier 1986, pp. 87-106. 
L'enquête ethnographique conduite actuellement, dans le cadre de postdoctorats à I'Institut de sciences sociales puis au sein du département d'anthropologie de l'Université de Tokyo ${ }^{15}$, porte avant tout sur la vie quotidienne et les pratiques politiques des résidents d'un quartier d'habitat social dans le Nord-Est de la capitale. La lutte conduite par un groupe de personnes installées depuis plusieurs années dans un parc des environs et confrontées fin 2012 à une procédure d'expulsion m'a amené à conduire en parallèle une observation participante parmi ce groupe et, au-delà, auprès de communautés de nojukusha de la zone.

Après les mois particulièrement tendus de la fin 2012, j'ai continué à être en lien constant avec les nojukusha rencontrés, en leur rendant visite une ou deux fois par semaine et en réalisant avec eux différents types d'activités. J'ai ainsi été initié par deux d'entre eux aux ficelles du métier de recyclage des canettes. Dans les arrondissements du nord-est de Tokyo, le recyclage des canettes et du papier est en effet une source régulière de revenus pour les nojukusha, à côté de travaux rémunérés plus ponctuels ${ }^{16}$. Les personnes dont il sera principalement question ici habitent dans deux «villages » de tentes, le premier dans un parc (une douzaine de tentes), l'autre en bord de rivière (moins de dix tentes) ${ }^{17}$. Tous sont des hommes, de plus de 50 ans pour la plupart, même si, à d'autres endroits, il y a aussi quelques femmes nojukusha. Leurs histoires de vie sont très variées : certains résident dans le quartier depuis une vingtaine d'années, d'autres sont arrivés à Tokyo après avoir tout perdu lors du tremblement de terre de mars 2011. Mais une majorité a été, et continue parfois à être, selon les opportunités trouvées, travailleur journalier. La transformation des mécanismes de recrutement des travailleurs journaliers, la raréfaction des emplois et le vieillissement ont souvent eu pour effet de rendre encore plus précaire leur existence. Les organisations militantes ou religieuses qui, historiquement, étaient présentes aux côtés des travailleurs journaliers se mobilisent aussi désormais auprès de ces travailleurs devenus nojukusha, en organisant régulièrement des soupes populaires ou des événements festifs, et en soutenant les nojukusha dans leurs luttes.

Une présence régulière et durable - plus de trois ans désormais, sur un total prévu de quatre ans - sur le terrain est associée à différentes techniques d'enregistrement et de conservation d'une mémoire de vie et d'enquête : tenue d'un journal de terrain détaillé sur ordinateur, notes prises sur le moment, notamment pour les éléments les plus difficiles à mémoriser (chiffres...), puis intégrées au journal, photos et vidéos, enregistrements audio dans certains cas, archivage des tracts et informations diffusées par les associations et groupes militants, ainsi que de pages web... Pour protéger l'anonymat des personnes évoquées, vis-à-vis des services municipaux mais aussi de manière plus générale, je ne précise pas le nom du quartier ou de l'arrondissement et j'omets ou modifie des détails qui pourraient permettre d'identifier les lieux ou les personnes.

15. Cette recherche a pu être conduite grâce au soutien de la Fondation Japon et de la Société japonaise pour la promotion de la science (JSPS).

16. Pour un panorama plus global, voir : Hours M., « La pauvreté urbaine au Japon. Réalités et représentations », Transcontinentales, $n^{\circ}$ 5, 2007, pp. 121-138; Hours M., "Les Nojukusha de Tokyo : relégation, déni de pauvreté et réponses parcellaires », in Bruneteaux P., Terrolle D. (dir.), L'Arrière-cour de la mondialisation : ethnographie des panpérisés, Broissieux, Éditions du Croquant, 2010.

17. Les tentes sont, à ma connaissance, toujours individuelles, ce qui n'exclut pas bien sûr la possibilité de recevoir des visites. 
À quoi renvoie l'expression «pratiques politiques » utilisée ici ? Le qualificatif « politique » a deux acceptions principales 18 , la première renvoyant à la sphère institutionnalisée de gouvernement et la deuxième au système social de rapports de pouvoir et à leur transformation. En ce deuxième sens, on peut définir les pratiques politiques comme des actes contribuant à transformer le système des rapports de pouvoir en un point ou plus globalement. Les pratiques politiques subalternes sont donc les actions permettant à leurs acteurs et actrices, collectivement ou individuellement, de se libérer de telle ou telle position subordonnée dans le réseau de relations de pouvoir.

Dans cette perspective, on peut observer pour les nojukusha et, au-delà, pour les habitants des quartiers populaires une série de pratiques politiques non-institutionnalisées qui sont autant de tactiques 19 pour contester, contourner ou éluder des rapports de pouvoir défavorables. À partir des notes prises à ce sujet les deux premières années (2012-2014), j’ai pu établir des parallèles entre différentes pratiques observées fonctionnant selon des dynamiques similaires et construire ainsi, en fonction de la manière dont elles font joner les rapports de pouvoir, une typologie 20 constituée de six types de pratiques : renversement d'un rapport de pouvoir, négociation, contournement, grignotage, création d'espaces autonomes plus ou moins temporaires et émancipation ${ }^{21}$. Pour tester la fécondité de cette typologie des pratiques politiques subalternes au-delà du contexte particulier de son élaboration - un quartier populaire de Tokyo -, je l'ai mobilisée comme grille de lecture du témoignage d'une ouvrière d'usine française 22 dans lequel j'ai retrouvé les six types de pratiques, à une ou plusieurs reprises, selon les types de pratiques, ce qui suggère que cette typologie peut prétendre à une validité au-delà du contexte dans lequel je l'utilise ici. Vu qu'il s'agit d'une typologie analytique, articulée autour des manières dont les subalternes « négocient » des rapports de pouvoir qui leur sont défavorables, elle doit pouvoir être mobilisée pour analyser des situations structurellement similaires dans des contextes historiques et géographiques variés.

Ces différentes pratiques politiques ont pour point commun d'être discrètes, voire à peine visibles, à l'exception de la première, la seule capable d'inverser un rapport de pouvoir et, de ce fait, la moins fréquente. Ce ne sont pas en général des actes que leurs acteurs mentionnent, et encore moins revendi-

18. Lefort C., Essais sur le politique : XIXe-XXe siècles, Paris, Seuil, [1986] 2001, p. 8, 19-20; Castells M., La Question urbaine, Paris, Maspero, [1972] 1981, p. 326.

19. De Certeau M., L'Invention du quotidien. 1, Arts de faire, Paris, Gallimard, [1980] 1990, pp. 60-63.

20. La typologie distingue à des fins d'analyse des types de pratiques qui, dans la réalité, sont souvent entremêlés.

21. Une première présentation de cette typologie a été faite lors d'une communication au Congrès mondial de l'Association sociologique internationale (Yokohama, juillet 2014).

22. Rosière S., Ouvrière d'usine : petits bruits d'un quotidien prolétaire, St-Georges d'Oléron, Éditions libertaires, 2010. 
quent. Et s'ils les évoquent, ils ne le font pas nécessairement en mobilisant le vocabulaire de la politique. Ce ne sont donc pas d'abord les acteurs, mais le chercheur qui qualifie ces pratiques de «politiques » à partir des critères de politicité explicités dans la définition des pratiques politiques proposée plus haut : est « politique » ce qui contribue à transformer des rapports de pouvoir, que les acteurs utilisent ou non ce qualificatif pour parler de leurs actes. Pour le dire autrement, la définition retenue est une définition matérialiste de l'action politique, et non une définition nominaliste : elle ne fait pas de la qualification des actes par leurs auteurs la condition sine qua non de leur politicité. Cette option matérialiste se traduit ici par l'accent mis sur la description et l'analyse des situations et des interactions, plutôt que sur la retranscription des paroles des acteurs, qu'il est au demeurant difficile de restituer avec une complète exactitude puisqu'il s'agit le plus souvent, sauf dans les cas où je dispose d'un enregistrement vidéo ou audio, de notes prises a posteriori et de mémoire.

Le subalterne est, par définition, pris dans un système de relations de pouvoir qui lui sont défavorables. De cette position subalterne, tactiques et stratagèmes peuvent être mis en œuvre pour tenter de se libérer de telle ou telle situation de domination, par le renversement d'un rapport de pouvoir, la négociation, le contournement ou le grignotage. Ces quatre tactiques sont explicitement ou implicitement relationnelles, elles sont élaborées dans un dialogue constant avec l'autre moitié de la relation de pouvoir. À l'inverse, les deux derniers types de tactiques - création d'espaces autonomes, émancipation - consistent à trouver des voies pour sortir de cet antagonisme visible ou souterrain, en construisant des positions autonomes en dehors de ces relations de pouvoir défavorables.

La première partie de cet article sera consacrée aux quatre premières tactiques, caractérisées par leur caractère dialogique, et la seconde aux deux dernières, qui constituent des tentatives de s'extraire d'un rapport de pouvoir défavorable. Ces différents types de pratiques constituent-ils des "résistances " ? Je montrerai en troisième partie que la mobilisation de ce schéma conceptuel s'avère fructueuse à condition d'opérer un distinguo entre résistance pour soi et résistance en soi. Ce distinguo permet en outre de rendre plus apparente la dynamique des rapports de pouvoir dont la résistance, telle qu'elle est communément entendue, ne constitue que le versant subalterne.

\section{Tactiques en vue d'une fin}

Parmi les six types de pratiques politiques repérées, les quatre premiers sont mobilisés par les nojukusha avec pour objectif une amélioration ou une défense de leurs conditions d'existence. Ils se développent dans le cadre d'une confrontation, ouverte ou évitée, avec l'autre de la relation de pouvoir, le choix d'une tactique, plutôt que d'une autre, dépendant notamment de l'état des forces en présence. 
Le premier type de pratiques est sans doute le plus étudié, étant aussi le plus visible. De fait, on pourrait considérer que c'est précisément le champ d'études de la sociologie des mouvements sociaux ${ }^{23}$. C'est le domaine de la "résistance dure », aux antipodes des pratiques plus discrètes de « résistance douce 24 » illustrées par les types suivants. Ce sont aussi, en dehors de circonstances extra-ordinaires, les pratiques les moins fréquemment observées. Ainsi, dans le cas des nojukusha, les exemples sont rares, mais mémorables. Ces événements font d'ailleurs désormais partie de la mémoire des luttes des travailleurs journaliers - nojukusha, mémoire entretenue par les militants, notamment par la projection plus ou moins régulière de documentaires consacrées à ces épisodes des luttes ${ }^{25}$. Les luttes victorieuses des nojukusha contre des tentatives d'expulsion participent du même type. La dynamique de renversement fonctionne d'une façon bien documentée par les spécialistes des mouvements sociaux : par différents moyens - nombre important de personnes mobilisées faisant face à la police ou l'administration, utilisation des médias pour dénoncer l'expulsion à venir... -, les nojukusha et les militants qui les soutiennent parviennent à inverser un rapport de force défavorable et à transformer la situation à leur avantage. Mais la plupart du temps, et précisément du fait de la difficulté à construire le pouvoir 26 nécessaire pour affronter et inverser un rapport de force défavorable, les subalternes mobilisent plutôt des tactiques à l'envergure plus modeste, comme la négociation, le contournement ou le grignotage. Les réseaux d'interdépendance et coopération qui structurent la vie sociale donnent aux différents groupes et personnes impliquées un pouvoir d'action sur les groupes et personnes avec lesquelles 27 elles sont en rapport d'interdépendance : les paysans peuvent cesser de distribuer les denrées qu'ils produisent, les ouvriers peuvent se mettre en grève. C'est ce que Frances Fox Piven et Richard Cloward nomment « perturbation institutionnelle » (institutional disruption) 28 ou « pouvoir de perturbation » (disruptive power) ${ }^{29}$, précisant que certaines personnes «sont parfois si isolées de toute forme de parti-

23. Voir par exemple : McAdam D., Freedom Summer, New York, Oxford University Press, 1988 ; Tilly C., Tarrow S., Contentious Politics, Boulder, Paradigm Publishers, 2007.

24. La distinction est de Motoji Matsuda (Urbanisation from Below: Creativity and Soft Resistance in the Everyday Life of Maragoli Migrants in Nairobi, Kyoto, Kyoto University Press, 1998, p. 22).

25. Voir, pour Tokyo (quartier de San’ya) : Satō M. [佐藤満夫], Yamaoka K. [山岡強一], Yamayararetara yarikaese [山谷-やられたらやりかえせ, Yama-Contre attaque], documentaire, 1985. Pour Osaka (quartier de Kamagasaki), voir : Kimu I. [金稔万], Kama no jūminhyō wo kaese! [釜の住民票を返せ!, Rendez-nous le certificat de résidence à Kamagasaki !], documentaire, 2008.

26. Un des mots d'ordre des mobilisations pendant l'Unité populaire au Chili (1970-1973) était d'ailleurs : «crear, crear, poder popular » (créer, créer, un pouvoir populaire).

27. J'applique, ici et plus loin, la règle de l'accord de proximité.

28. Piven F.F., Cloward R.A., Poor People's Movements: Why They Succeed, How They Fail, New York, Vintage books, [1977] 1979, pp. 24-31.

29. Piven F.F., Challenging Authority: How Ordinary People Change America, Lanham, Rowman \& Littlefield, 2006, p. 5. 
cipation institutionnelle significative que la seule "contribution" à laquelle elles peuvent mettre fin est leur apport au calme de la vie civile; ils peuvent initier une émeute 30 ». De fait, les deux mobilisations ayant fait l'objet des documentaires cités plus haut ont pris la forme d'émeutes (1983-1984 à San'ya, arrondissement de Taito, Tokyo et 2007 à Kamagasaki, arrondissement de Nishinari, Osaka). La situation sociale marginale des nojukusha et, comme on va le voir, les formes de vie relativement autonomes qui sont les leurs les privent en revanche d'une partie du « pouvoir de perturbation » offert par l'interdépendance.

Comment est-il possible d'améliorer son sort ou d'échapper à des situations de domination quand on ne dispose pas de la puissance nécessaire à l'affrontement direct ? Comment le faible peut-il parfois triompher sur plus puissant que lui ? À défaut d'avoir pour soi la force ou le pouvoir requis, on peut tenter de mobiliser la ruse et l'astuce - la mètis ${ }^{31}$. Si le pouvoir forme un bloc, la mètis est, elle, "multiple et diverse 32 ", et les combines s'élaborent de manière pragmatique en fonction des opportunités ${ }^{33}$, des points faibles et des angles morts de l'adversaire.

\section{Négociation}

La négociation offre une bonne illustration du genre de tactiques mobilisées. Pour de nombreux nojukusha de Tokyo, et au-delà, la collecte de canettes usagées, de cartons, de journaux et de magazines constitue une source importante de revenus. Canettes et papier sont vendus à des entreprises qui paient au kilo, les prix variant selon les entreprises et le cours du marché - de l'aluminium, pour ce qui est des canettes. Le prix de l'aluminium a augmenté en 2013 et 2014, avant de baisser en $2015^{34}$; la vente rapportait en avril 2015 environ 155 yens par kilo (soit 1,15 euro), 127 yens fin juillet 2015, 114 yens début septembre 2015 - contre 108 yens en avril 2013 mais 170 en décembre 2014 - dans une des entreprises ayant le double avantage de pratiquer des prix intéressants et de se situer dans la même partie de la ville ${ }^{35}$.

En juin 2013, un élu de l'assemblée d'arrondissement a enrôlé des journalistes d’une chaîne de télévision privée pour réaliser une émission sur le travail

30. Piven F.F., Cloward R.A., Poor People's Movements, op. cit., p. 25.

31. Detienne M., Vernant J.-P., Les Ruses de l'intelligence : la mètis des Grecs, Paris, Flammarion, [1974] 2008, p. 19.

32. Ibid., p. 25.

33. De Certeau M., L'Invention du quotidien. 1, Arts de faire, op. cit., pp. 60-61.

34. Il s'agit d'une baisse assez générale du prix des matières premières qui touche tout autant les États fortement tributaires de leurs exportations de biens du secteur primaire, comme l'Équateur ou le Venezuela, que les nojukusha...

35. Les données concernant l'évolution du prix du kilo d'aluminium ont été obtenues, selon les périodes citées, soit directement du fait de ma présence lors des ventes des nojukusha à l'entreprise, qui leur délivre alors des reçus, soit par les récits des recycleurs, pour qui la variation des cours, qui affecte directement leurs revenus, est une question importante. 
réalisé par les recycleurs de canettes et le dénoncer comme du vol. L'argument avancé était que l'arrondissement avait investi des sommes importantes dans son programme de recyclage et que les recycleurs détournaient une partie des ressources à leur profit au détriment du programme municipal. L'évaluation des gains des recycleurs et, par voie de conséquence, des pertes de l'arrondissement était largement exagérée, comme le reconnaîtraient les fonctionnaires municipaux lors de la réunion décrite ci-dessous.

Les recycleurs, accompagnés de militants, rendirent alors visite aux services de l'arrondissement chargés de la collecte des déchets pour leur poser une série de questions (juillet 2013). Durant cette rencontre ${ }^{36}$, la négociation est constante, tant sur le nombre de personnes admises à l'intérieur du bâtiment, par exemple, que sur la qualification de l'activité de recyclage de canettes par des individus. Je vais commencer par décrire rapidement la dynamique de la réunion avant de revenir sur les tactiques utilisées. La négociation concerne d'abord des aspects très concrets qui vont définir les conditions de la rencontre : temps et espace octroyé, nombre de participants. Recycleurs et militants d'un côté, fonctionnaires de l'autre, savent que ces éléments déterminent le type de rapport de force qui va être établi et essaient donc de créer des conditions qui leur soient favorables. Et s'il arrive que des agents de l'administration n'aient pas conscience de ce type d'enjeu, une seule rencontre entre deux ou trois fonctionnaires et un groupe de vingt à trente personnes suffit en général à la prise de conscience. Dans une autre situation de négociation observée, après une première réunion en très fort déséquilibre numérique, les agents de l'administration sont, la fois suivante, revenus avec un groupe d'une vingtaine de personnes « pour faire bon poids».

Lors de la rencontre qui nous intéresse, les fonctionnaires ont commencé par déclarer : "vous êtes trop nombreux, pouvez-vous choisir quelques personnes pour venir au guichet ? ». Les recycleurs répondirent qu'ils étaient tous concernés et qu'ils voulaient donc tous entrer, mais qu'ils se mettraient sur le côté si une autre personne venait au guichet. Cette fois-ci, ce sont eux qui gagnèrent cette manche de la négociation, ce qui leur permit d'imposer un rapport de force très différent de celui qui se serait établi si seul un petit groupe avait pu entrer. Sur ce point, négociation et renversement du rapport de pouvoir se rejoignent : un groupe important est en mesure d'imposer un rapport de force potentiellement plus favorable qu'un groupe plus réduit. En d'autres occasions, les nojukusha ont perdu la première manche de la négociation et dû se résigner à envoyer seulement trois représentants à l'intérieur des bâtiments. Il est intéressant de noter que la négociation s'inscrit dans la durée : confrontés aux visites récurrentes d'un groupe important, les fonctionnaires s'organisent progressivement pour restreindre l'accès des bâtiments aux quelques per-

36. La description qui suit est issue des notes prises dans mon journal de terrain. Un enregistrement vidéo a aussi été réalisé. 
sonnes autorisées, en rassemblant de leur côté un nombre important d'agents de la mairie d'arrondissement pour physiquement «faire barrage » devant l'entrée du bâtiment.

Un des points de la discussion ce jour-là portait sur les sommes d'argent évoquées dans le reportage télévisé - celles investies par la mairie et celles gagnées par les recycleurs. Le groupe considérait que ces chiffres avancés étaient faux et obtinrent sur ce point confirmation de la part des fonctionnaires. L'échange se poursuit alors sur l'accusation principale de l'élu dans le reportage : que l'activité des recycleurs est un vol. Les agents de la mairie finirent par affirmer de façon claire que la collecte des canettes et journaux n'était pas permise, mais qu'ils ne la considéraient pas comme un vol. Pendant la discussion, les positions avancées s'appuient sur des arguments de deux types, logiques et moraux. Un des recycleurs fait par exemple valoir que, dans un quartier voisin, un groupe de femmes recycle aussi les canettes et que personne ne les a traitées de voleuses - argument logique. Tous les recycleurs expriment leur colère d'être traités de voleurs alors qu'ils travaillent dur pour gagner leur vie et survivre : «ce n'est pas juste ». Est mise en avant ici la revendication d'un « droit de vivre » comme «premier droit naturel », «préalable et supérieur à tout autre droit public et privé 37 ».

De fait, la négociation entre les différentes autorités locales, tant à propos de l'occupation de l'espace public que sur des sujets plus circonstanciels, s'appuie fortement sur l'idée des droits humains comme revendication morale d'un niveau supérieur aux autres considérations. Le jeune Karl Marx mobilisait le même type d'arguments contre l'une des lois discutées à la Diète rhénane à l'automne 1842, qui projetait de punir le ramassage de bois mort d'une peine de travaux forcés, alors que c'était jusqu'alors un droit d'usage. Un des motifs énoncés lors des débats était que les paysans pauvres pratiquaient parfois des entailles dans les jeunes arbres pour les « aider à mourir » et pouvoir alors les couper. Dans sa série d'articles consacrés aux «Débats sur la loi relative au vol de bois 38 ", Marx dénonce ainsi la tentation de la Diète de "faire céder le pas [...] au droit des hommes devant celui des jeunes arbres 39 ». Dans les deux cas - «vol » de bois et « vol » de canettes - les enjeux sont identiques ; leurs détracteurs mettent en avant les pertes financières occasionnées par les « vols » tandis que défenseurs ou acteurs leur opposent un « droit de

37. Nicolas J., La Rébellion française : mouvements populaires et conscience sociale : 1661-1789, Paris, Gallimard, [2002] 2008, p. 424.

38. Marx K., " Débats sur la loi relative au vol de bois (octobre-novembre 1842) », in Bensaid D., Les Dépossédés: Karl Marx, les voleurs de bois et le droit des pauvres, textes traduits par Lascoumes P., Zander H. et Poirier J.-F., Paris, la Fabrique, 2007, pp. 91-119. Cette traduction des articles de la Gazette rhénane est issue d'une édition critique antérieure, désormais épuisée (Lascoumes P., Zander H., Marx K., Du "Vol de bois » à la critique du droit : Karl Marx à la "Gazette rhénane ", naissance d'une méthode. Édition critique de "Débats sur la loi relative an vol de bois" et "Justification du correspondant de la Moselle ", traduit par Renouf L. et Zander H., Paris, PUF, 1984).

39. Marx K., "Débats sur la loi relative au vol de bois », op. cit., p. 93-94. 
(sur)vivre ». Mais au-delà de la dispute en elle-même, ce qu'il faut noter ici, c'est que les arguments moraux constituent un élément central de la pratique politique subalterne de négociation, et ce précisément parce que ce type d'arguments doit en principe être pris en compte quand bien même il est formulé à partir d'une position subalterne : «si vous prétendez respecter les droits humains, comme vous le déclarez sur le site internet de la mairie d'arrondissement, alors vous devez prendre en compte nos revendications... 40 » Recycleurs et militants concluent la discussion en déclarant que si quelque chose d'autre arrive, ils viendront à nouveau. Ce à quoi les fonctionnaires répondent : «mais ne venez pas aussi nombreux », avant de s'entendre répliquer : «nous viendrons tous car nous sommes tous concernés »...

Les détails donnés ici montrent d'abord que la négociation est constante, du début jusqu'à la fin de la rencontre. Ils montrent aussi que deux types d'armes sont mobilisées. Les arguments moraux, tout d'abord, constituent de puissantes «armes des faibles 41 » : ils peuvent en principe être avancés indépendamment du statut social ou de la position dans le système des rapports de pouvoir car ils font appel à des valeurs supposées partagées par tous. Mais ce sont des armes puissantes seulement dans la mesure où la partie située du "bon côté » du rapport de force décide de "prendre les droits au sérieux 42 », c'est-à-dire comme quelque chose de plus que de simples droits formels. Pour cette raison, et afin d' ' encourager » le respect des droits, l'utilisation combinée d'une autre arme - la construction, temporaire ou plus durable, d'un pouvoir collectif - s'avère très utile. Comme indiqué plus haut, la négociation est rendue plus aisée si elle est associée à un certain degré de confrontation. Dans le cas présenté ici, un syndicat de recycleurs de canettes et papier a été créé il y a quelques années et c'est en tant que syndicat que les recycleurs ont rendu visite à la mairie d'arrondissement.

Si le renversement des rapports de pouvoir et la négociation sont, à des degrés différents, associés à une confrontation, le « contournement » et le « grignotage » tendent, à l'inverse, à éviter la confrontation.

\section{Contournement}

Quand un obstacle ne peut être surmonté, on peut essayer plutôt de le contourner. De même, face à des rapports de pouvoir qu'on ne peut pas espérer transformer ou renverser, il est parfois possible de trouver des manières de les contourner. En 2012, au moment de mon arrivée sur les lieux, le groupe de nojukusha qui résident dans le parc - certains depuis 20 ans - est confronté à une nouvelle procédure d'expulsion menée par les services de la mairie res-

40. Argument avancé de manière récurrente lors des négociations des nojukusha avec des fonctionnaires municipaux.

41. Scott J.C., Weapons of the Weak, op. cit.

42. Dworkin R., Taking Rights Seriously, Cambridge, Harvard University Press, 1977. 
ponsables de l'administration des parcs et cours d'eau qui conduisent une opération de rénovation du parc. En 2012, un nouvel arrêté d'expulsion est placardé à l'entrée du parc, demandant le départ des nojukusha avant la date indiquée. Les mobilisations des personnes concernées, accompagnées de militants et de sympathisants, ne parviennent ni à inverser le rapport de force ni à trouver une issue négociée. Un matin, des dizaines de fonctionnaires, agents de sécurité et policiers arrivent dans le parc pour procéder à l'expulsion. Comme les tentes des nojukusha avaient été déplacées à vingt mètres de l'endroit où l'expulsion devait avoir lieu, celle-ci n'eut pas lieu, mais des palissades de deux mètres de haut furent construites autour du coin du parc où un nouveau village de tentes avait été édifié, l'isolant ainsi du reste du parc. Comme on va le voir, c'est le recours à une tactique de contournement qui a permis d'empêcher les services de la mairie de procéder à l'expulsion des nojukusha.

Le parc où les tentes étaient construites relève en majeure partie de la compétence de l'arrondissement, mais ses franges, le long des immeubles privés qui bordent le parc relèvent de la compétence de la métropole (Tōkyō-to). C'est à cet endroit que les tentes avaient été déplacées. Les services de la mairie d'arrondissement n'avaient donc pas, légalement, le pouvoir de procéder à une expulsion d'un espace ne relevant pas de leur compétence. Nojukusha et militants ont avancé, en outre, un autre argument logique : derrière les immeubles longeant le parc ont été construits, sans autorisation municipale, plusieurs abris utilisés par les habitants des immeubles et les entreprises pour stocker du matériel ou des outils. Ils ont fait valoir qu'il serait injuste que les tentes nouvellement construites soient détruites sans que le soient aussi les abris situés dans la même zone du parc... À y regarder de plus près, les arguments logiques évoqués ici et précédemment sont en même temps des arguments moraux : si l'égalité est bien, comme le discours public le prétend, une valeur sociale cardinale, il ne serait pas juste que deux situations similaires soient traitées de manière différente. Le jour de l' « expulsion », fonctionnaires et agents de sécurité durent faire face aux habitants du parc qui, avec l'aide d'une soixantaine de sympathisants et de militants, formaient une chaîne humaine pour protéger les tentes nouvellement construites. La confrontation était donc aussi présente, mais, sans le recours au contournement, elle aurait sans doute été inutile.

\section{Grignotage}

Le grignotage est une autre tactique qui évite la confrontation, en essayant de passer la plus inaperçue possible. Ce qui ne peut pas être obtenu au grand jour, par la confrontation ou par des moyens légaux peut potentiellement être grignoté petit à petit - qu'il s'agisse d'espace, de ressources ou d'évasion fiscale... Son efficacité discrète en fait probablement la pratique politique subalterne la plus utilisée, permettant d'obtenir différents types de gain sans le coût 
potentiellement élevé du couple confrontation-répression ${ }^{43}$. Ses différentes facettes ont été largement documentées, du braconnage dans l'Angleterre du début du XVIII siècle 44 aux stratagèmes des paysans japonais de l'ère Tokugawa (1600-1868) pour échapper à la taxe sur le riz ${ }^{45}$, des occupations de terrains dans le Chili de la deuxième moitié du XX siècle ${ }^{46}$, à la " politique du ventre » dans le Zaïre de Mobutu 47, ou à l' " avancée silencieuse, durable et multiple des gens ordinaires sur les possédants, les puissants ou le public 48 » dans les villes du Moyen-Orient. Le grignotage est, en temps normal, une tactique silencieuse, mais si par hasard les «coupables »sont amenés à donner leur point de vue sur la question, il est potentiellement associé à des revendications morales mettant en avant le «droit de vivre », la nécessité, ou même le droit à un accès égal aux ressources naturelles ou publiques, comme dans le cas des braconniers anglais condamnés à la pendaison pour avoir abattu un cerf : « ils pouvaient difficilement être convaincus que le crime pour lequel ils souffraient méritait la mort. Ils dirent que les cerfs étaient des bêtes sauvages et que les pauvres, autant que les riches, avaient le droit de les utiliser 49 ».

Le grignotage est aussi une tactique largement utilisée par les nojukusha de Tokyo, particulièrement pour ce qui a trait à l'occupation des espaces publics. Une partie de leurs activités de recyclage, situées à la frontière floue de ce qui est permis et ce qui ne l'est pas, peut aussi être considérée comme du grignotage : la collecte de canettes n'est pas clairement autorisée, mais elle est tolérée, comme l'était la collecte de bois mort dans la Rhénanie du XXe siècle ${ }^{50}$. Le grignotage, qui se déploie dans la durée, est par nature instable ${ }^{51}$, avec des avancées et des reculs. En avril 2014, quelques nojukusha profitent de travaux en cours et de l'érection de barrières pour se réinstaller sous une autoroute aérienne, endroit qu'ils avaient dû quitter auparavant. À cause des barrières et des installations du chantier, ils ne peuvent pas construire des tentes comme d'habitude mais ils arrivent cependant à s'installer. Un mois plus tard, les résidents sont désormais au nombre de dix. À mesure que le temps passe et que le nombre de personnes augmente, il devient plus difficile d'intervenir pour les services de la ville. Les nojukusha le savent, mais les fonctionnaires le savent aussi. À un autre endroit, sous une autre autoroute aérienne, il y avait

43. Scott J.C., Weapons of the Weak, op. cit., p. 299 ; Scott J.C., Domination and the Arts of Resistance, op. cit., p. 190.

44. Thompson E.P., Whigs and Hunters: The Origin of the Black Act, Harmondsworth, Penguin, [1975] 1977.

45. Watanabe H., A History of Japanese Political Thought, 1600-1901, traduit par Noble D., First English, I-House Press, 2012, p. 227.

46. Garcés M., Tomando su sitio: el movimiento de pobladores de Santiago, 1957-1970, Santiago, LOM, 2002.

47. Bayart J.-F., L'État en Afrique : la politique du ventre, Paris, Fayard, [1990] 2006, p. 291-292.

48. Bayat A., Life as Politics, op. cit., p. 56.

49. Cité par Thompson E.P., Whigs and Hunters, op. cit., p. 162. Je souligne.

50. Marx K., «Débats sur la loi relative au vol de bois », op. cit.

51. Sur l'instabilité et l'incertitude inhérents aux tactiques et à la mètis, voir : Detienne M., Vernant J.-P., Les Ruses de l'intelligence, op. cit., p. 21 ; Certeau M., L'Invention du quotidien 1, op. cit., p. 61. 
auparavant un petit village de tentes. À l'occasion d'une autre opération de rénovation, les services de la mairie avaient obtenu le départ de tous les résidents, sauf un, en leur offrant notamment un logement gratuit pendant un an. Quand deux nouvelles tentes apparurent sur les lieux pendant la nuit, elles furent détruites le lendemain par des ouvriers envoyés par l'arrondissement. Le grignotage est une tactique bien connue des nojukusha comme des fonctionnaires...

Ce qui fait du grignotage une tactique efficace pour les subalternes, c'est, en plus de la discrétion de sa progression mesurée et silencieuse, l'argument moral opposé implicitement ou explicitement à l'autre «camp ». Les nojukusha qui, par nécessité, s’installent dans les parcs peuvent légitimement revendiquer un droit humain fondamental, le droit de vivre, voire un « droit à la ville 52 » égal à celui des autres citadins. Quoi qu'il en soit, et comme cela a déjà été noté plus haut, la légitimité de cette revendication morale ne la rend pas, en soi, capable de s'opposer avec succès à toute tentative d'expulsion. Mais elle représente une alliée précieuse des tactiques de grignotage. De même, si le grignotage, comme les autres tactiques, ne sont pas une prérogative exclusive des subalternes, la légitimité morale de ce type de pratiques est plus clairement présente dans leur cas que dans les autres, du fait précisément de leur position sociale.

Le renversement des rapports de pouvoir est en général ponctuel, parfois un peu plus durable. Les nojukusha ne parviennent à inverser les rapports de force que dans des situations de confrontation spécifiques et ce renversement d'un rapport de pouvoir particulier n'est pas une fin en soi, mais un moyen en vue d'une fin. Il s'agit de redresser un tort, d'améliorer une situation perçue comme injuste et insupportable, qui va à l'encontre de certains besoins de base ou même remet en cause la possibilité de vivre dignement. Négociation, contournement et grignotage cherchent de même à obtenir un gain dans le cadre de rapports de pouvoir en cours, en mobilisant d'autres moyens que l'affrontement direct.

\section{Quand le moyen est aussi la fin : autonomies en construction}

La construction d'espaces autonomes est, à l'inverse, une fin en soi : le moyen est la fin. On cherche à s'extraire de rapports de pouvoir défavorables pour atteindre une forme d'autonomie, toujours relative. L'émancipation est de même un moyen et une fin. Dans les quartiers populaires, la domination la plus fortement ressentie est sans doute la domination économique ${ }^{53}$. La rareté des ressources monétaires délimite en grande partie ce qui est possible et ce

52. Lefebvre H., Le Droit à la ville, Paris, Anthropos / Economica, [1968] 2009 ; Harvey D., Rebel Cities: From the Right to the City to the Urban Revolution, New York, Verso, 2012, p. 3-26.

53. Dans des contextes politiques relativement démocratiques. 
qui ne l'est pas. Partant, la construction d'espaces autonomes constitue souvent une tentative pour se libérer de certaines de ces dépendances économiques.

\section{Construction d'espaces autonomes}

Nous sommes, pour une large part d'entre nous, né-e-s en ville et les conditions de la vie urbaine sont devenues souvent à ce point transparentes qu'on ne les perçoit plus. Vivre en ville signifie communément d'avoir à payer - ou d'avoir payé - pour le logement dans lequel nous vivons, la nourriture que nous consommons, l'eau que nous buvons, les transports que nous utilisons, l'électricité et le gaz que nous dépensons... Il y a de fait un lien pour ainsi dire consubstantiel entre ville et marché, lien dont les nouveaux citadins, tout juste arrivés de zones rurales africaines ${ }^{54}$ ou latino-américaines 55 font l'expérience avec le plus d'acuité, en Persans d'un nouveau genre. Ils opèrent en effet une transition accélérée et souvent douloureuse entre des formes de vie relativement autonomes - ayant pour base le travail de la terre, et dans lesquelles le marché joue un rôle réduit -, et des modalités d'existences radicalement hétéronomes, avec l'argent comme médiation principale.

Comment les nojukusha s'inscrivent-ils dans ce panorama ? Ils ne paient tout d'abord ni loyer, ni eau ni électricité et ils utilisent rarement les transports publics. Ils installent des tentes dans des parcs, au bord des rivières, parfois sous des autoroutes aériennes qui les protègent de la pluie, c'est-à-dire dans des espaces publics non bâtis. Ils choisissent de préférence des endroits proches de toilettes publiques et de robinets d'eau. Dans certains cas, ils bénéficient aussi de l'éclairage public des rues ou des parcs. Leur utilisation des espaces et infrastructures publiques ne coûte pas grand-chose à la ville : l'éclairage public fonctionne qu'ils soient présents ou non, les toilettes sont utilisées par les passants et nettoyées de toute façon par des agents d'entretien. Ils utilisent clairement plus d'eau que les passants ne le font, pour boire, cuisiner ou laver, mais comme l'eau doit être utilisée près des robinets, ou transportée, sa consommation est relativement limitée et constitue un supplément négligeable de dépense dans les budgets des arrondissements. Pour se laver, ils peuvent utiliser les bains publics (sentō) dont ils paient l'entrée, à moins qu'ils n'aient obtenu des tickets auprès d'associations de soutien. Ils font ainsi un usage

54. Matsuda M., Urbanisation from Below, op. cit., p. 318.

55. Contreras K., Pérez R., Pickard M., Rivera A., Zunino M., « Ciudades rurales sustentables, despojo y contrainsurgencia en Chiapas ", in Sandoval Palacios J.M., Álvarez de Flores R., Fernández Moreno S.Y. (coord.), Planes geoestrategicos, desplazamientos y migraciones forzadas en el área del proyecto de desarrollo e integración de Mesoamérica, Medellín / Mérida / México, Universidad de Antioquia, Departamento de Trabajo Social, Facultad de Ciencias Sociales y Humanas / Centro de Estudios de Fronteras e Integración, Universidad de los Andes / Seminario Permanente de Estudios Chicanos y de Fronteras, DEAS-INAH, 2011, p. 150-151. Traduction française : Contreras K., Pérez R., Pickard M., Rivera A., Zunino M., «Villes rurales durables, spoliation et contre-insurrection au Chiapas », DIAL, $\mathrm{n}^{\circ} 3331 \&$ $3333,2015$. 
plein des infrastructures urbaines dont le coût de maintenance est à peu près le même qu'ils les utilisent ou non.

Les recycleurs collectent canettes, cartons et journaux en se déplaçant en général à vélo, parfois à pied - notamment dans le cas de personnes relativement âgées ou à l'équilibre précaire -, avec souvent dans ce cas un chariot où ils empilent ce qu'ils récupèrent. Les vélos de recycleurs se reconnaissent aisément à la grille ajoutée sur le porte-bagage pour gagner en superficie et transporter plus de sacs de canettes ou de piles de journaux... Comparés à des formes plus courantes de vie urbaine, les nojukusha ont donc, effet de la nécessité, des formes de vie relativement autonomes, dans lesquelles la médiation de l'argent joue un rôle beaucoup plus réduit. Ils ne sont pas, bien sûr, en dehors de la société marchande et leurs revenus dépendent, comme indiqué plus haut, de l'évolution des prix du marché (aluminium, papier). Mais ils vivent à la marge de la société marchande - de la société urbaine -, et construisent, par nécessité, des espaces d'autonomie relative.

Un de ces espaces d'autonomie surgit des pratiques agricoles urbaines présentes chez les nojukusha, comme aussi d'ailleurs dans les quartiers avoisinants. Les nojukusha ne cultivent pas systématiquement la terre, mais quand les lieux s'y prêtent - parcs, bords de rivière -, certains le font, avec plus ou moins de constance. Dans les endroits où sont installés des villages de tentes, si agriculture il y a, c'est en général le fait d'une ou deux personnes, les autres personnes ne participant que très peu, ou pas du tout. Dans les deux villages de tentes que j'ai pu observer, l'un dans un parc, l'autre en bord de rivière, sont associées cultures d'hiver - daikon (gros radis blanc), choux chinois, radis, légumes à feuilles (komatsuna, shungiku) -, cultures d'été - concombres, aubergines, poivrons, piments, haricots verts, maïs, pastèques et kin uri, une variété de melon - et parfois quelques fleurs - tulipes, volubilis (asagao), tournesols.

En quoi ces pratiques agricoles contribuent-elles à former un espace autonome ? Tout d'abord, pour leur alimentation comme pour les autres aspects de leur existence (logement, accès à l'eau...), les nojukusha sont contraints de trouver des voies alternatives pour subvenir à leurs besoins, car leur accès aux solutions marchandes dominantes est plus restreint que celui de leurs concitoyens ${ }^{56}$. Ce faisant, leur existence prend, sous l'effet de la nécessité, une tournure plus autonome que celle de la plupart des autres citadins puisqu'elle ne dépend pas, ou qu'elle dépend moins, d'une série d'intermédiaires marchands. Cette autonomie est doublement relative. Elle est relative d'abord parce que leurs revenus dépendent, je l'ai dit, du prix de l'aluminium sur le marché international et de la tolérance des autorités vis-à-vis de leurs activités de recyclage.

56. C'est la même chose pour les meubles et les ustensiles de cuisine qui sont le plus souvent des biens récupérés. 
Elle est relative ensuite puisque leur autonomie est à la fois plus grande, paradoxalement, que celle de leurs voisins, mais plus réduite que, par exemple, celle de paysans vivant en autarcie relative.

Ces pratiques agricoles en entraînent d'autres, tout particulièrement des pratiques de don - dons de plants, de graines ou de bulbes entre villages de tentes, dons de légumes et de fruits. Dans le village de tentes ayant survécu à la procédure d'expulsion évoquée, la majeure partie des cultures est devenue en quelque sorte le domaine réservé d'un des résidents qui s'en occupe presque seul et peut décider ce qu'il souhaite faire de sa production. Pour lui, comme pour l'autre cultivateur du village, la première destination des légumes et fruits récoltés est, avant même la consommation personnelle, le don et, plus précisément, le don à des personnes extérieures au village de tentes, comme c'est le cas des sympathisants. Ces dons, qui viennent en contrebalancer d'autres don de temps des gens venus soutenir les luttes... - constituent une manière non-marchande de s'efforcer de maintenir une forme d'équilibre dans l'échange des biens matériels et symboliques.

La construction d'espaces autonomes peut aussi naître d'occupations de terrains plus larges, comme celles conduites par le Mouvement des sans-terre (MST) au Brésil 57 ou les tomas chiliennes des années 1960 et 1970 58. Chaque fois, le contrôle physique de l'espace - qui peut être très temporaire - semble être un facteur décisif pour la mise en place d'espaces autonomes. Dans les villes du Moyen-Orient, les pratiques de grignotage des classes populaires poursuivent, si l'on en croit Asef Bayat, un double but de redistribution des biens sociaux et des opportunités et de construction d'autonomies ${ }^{59}$. Comme pour les nojukusha, ce deuxième objectif est contrebalancé par un besoin de sécurité 60 . De par l'instabilité des positions, la construction d'espaces autonomes est souvent temporaire et, comme pour la plupart des autres tactiques subalternes, ce qui est gagné aujourd'hui peut être perdu demain... C'est ce qui fait de l'émancipation une pratique politique à part dans la typologie proposée.

\section{Émancipation}

On peut définir les pratiques émancipatrices comme des pratiques permettant d'échapper - ou d'aider à échapper - à une forme ou une autre de rapports de subordination. Structurellement similaires à la construction d'auto-

57. Bleil S., Vie et lutte des Sans Terre au sud du Brésil: une occupation au Parana, Paris, Karthala, 2012.

58. Garcés M., Tomando su sitio, op. cit.

59. Bayat A., Life as Politics, op. cit., pp. 59-60.

60. «Dans leur quête de sécurité, les pauvres urbains sont en constante négociation et hésitation entre autonomie et intégration. Et ils continuent néanmoins à rechercher l'autonomie dans tous les espaces disponibles au sein des structures et des processus d'intégration. » (Ibid., p. 60.) 
nomies, les pratiques émancipatrices en diffèrent par leur temporalité. En somme, l'émancipation est une autonomie durable, une autonomie associée à une certaine forme de sécurité. Pour cette raison, ce type de pratiques est, avec le renversement des rapports de pouvoir, la pratique politique subalterne la moins fréquemment observée. De fait, les pratiques émancipatrices permettent, au moins pour un rapport de pouvoir en particulier, de cesser d'être dans une position subalterne.

Pour ce qui est des nojukusha de Tokyo, et même si l'émancipation est un processus se déployant sur le long terme et qu'il est donc difficile d'en parler autrement qu'a posteriori, ce que j'ai pu observer prend surtout la forme de projets de pratiques émancipatrices. Dans le village ayant échappé à la procédure d'expulsion, les deux personnes impliquées de manière régulière dans le jardinage ont toutes les deux évoqué, à des moments différents et alors que l'autre jardinier n'était pas présent, qu'elles aimeraient essayer de cultiver une parcelle de terre dans le département voisin, moins urbanisé. Un des deux jardiniers est même parti en reconnaissance à vélo mais il n'a, jusqu'à présent, pas poussé plus avant : il n'est pas allé discuter avec les agriculteurs âgés qui sont nombreux dans cette zone, même s'il a exprimé qu'il souhaitait le faire. En écho à ce qui a été noté plus haut du lien entre travail de la terre et autonomie, il est intéressant de noter que, pour ce nojukusha jardinier, l'émancipation semble passer par l'accès à la terre.

De fait, si on élargit la focale, le lien entre agriculture et autonomie, accès à la terre et émancipation forme le socle de pratiques politiques et luttes sociales variées, comme en témoignent la circulation du slogan « Terre et Liberté » des populistes russes Narodniki aux révolutionnaires mexicains puis espagnols, ou l'importance du thème de la réforme agraire dans l'Amérique latine de la seconde moitié du XXe siècle et au-delà, en Chine notamment. À l'inverse, les travaux de Karl Polanyi 61, parmi d'autres, ont bien montré le lien de cause à effet entre restriction de l'accès à la terre, enclosures et prolétarisation : privées d'accès aux terres communales qui assuraient une partie de leur subsistance et réduites « à choisir entre mourir de faim ou offrir [leur] travail sur le marché au prix qu'[elles] pourrai[ent] en tirer 62 », des fractions importantes de la population rurale anglaise viennent alors combler les besoins en main-d'œuvre pressants des manufactures en plein essor, vivant et travaillant désormais dans des conditions de radicale hétéronomie.

61. Polanyi K., The Great Transformation, Boston, Beacon Press, [1944] 2001, pp. 172-173, 187189. Voir aussi, pour le cas particulier français : Noiriel G., Les Ouvriers dans la société française : XIX $X^{e}-X X^{e}$ siècle, Paris, Seuil, [1986] 2002, p. 297-312.

62. Polanyi K., The Great Transformation, op. cit., p. 172. 


\section{Pratiques politiques et formes de résistance}

Les pratiques politiques décrites peuvent-elles être considérées comme des formes de résistance ? La définition proposée des pratiques politiques subalternes comme des « actions permettant à leurs acteurs et actrices, collectivement ou individuellement, de se libérer de telle ou telle position subordonnée dans le réseau de relations de pouvoir » repose sur le même schéma conceptuel dual que l'idée de résistance entendue comme autre de la domination. S’il y a une homologie structurelle entre les deux concepts, la notion de pratique politique ayant reçu une définition matérialiste, elle évacue du même coup le problème de l'intentionnalité, qui se pose pour la notion de résistance. Pour cette dernière, une chose est claire, si résistance il y a, c'est toujours une résistance $\grave{a}$, une résistance contre : s'il n'y a plus de force antagoniste, il n'y a plus non plus de résistance. La résistance est un concept relationnel.

\section{Résistant-e-s, résisté-e-s, observateurs et observatrices}

Pour cette raison, renversement du rapport de pouvoir et négociation, les deux pratiques repérées qui supposent une confrontation, sont très naturellement reconnues comme des formes de résistance : les nojukusha résistent, parfois avec succès, à une procédure d'expulsion et à la diffusion de représentations négatives de leur activité de recyclage, avec les conséquences que cela pourrait avoir sur leur quotidien. L'intentionnalité est évidente. Le contournement et le grignotage sont plutôt des pratiques qui évitent la confrontation, mais ce sont toujours des pratiques relationnelles : il y a en quelque sorte un dialogue, verbal ou non, entre les deux forces en présence. Là aussi, on peut y voir des formes de résistance, non pas directes, mais détournées, " par la bande ». Mais les choses sont déjà nettement moins claires : est-ce que construire une tente dans un espace public est un acte de « résistance " ? À quoi « résistent» les braconniers anglais décrits par E. P. Thompson ? Faut-il considérer que les efforts pour se procurer ce qui est nécessaire à la (sur)vie mettent en jeu une intention de résistance, et si oui, à quoi, contre quoi ou contre qui ? Considérer que les nojukusha ou les braconniers cherchent à résister « aux intempéries ", ou « à la faim » ne paraît pas très concluant, car on sort alors du schéma conceptuel opposant résistance et domination, comme dans l'acception parallèle d'une résistance « à la maladie ». Ce genre de questionnement semble ainsi conduire à une impasse.

Pour les pratiques construisant des espaces autonomes, la question n'a pas beaucoup de sens non plus : à qui ou à quoi résistent les nojukusha jardiniers ? Pour sortir de l'impasse, il importe d'opérer un changement d'échelle pour replacer la résistance dans le triangle d'acteurs qui la constitue - résistant-e-s, résisté-e-s, observateurs ou observatrices - car, comme le notent Anshuman 
et Pushkala Prasad 63, la résistance, intentionnelle ou non, est aussi un phénomène qui existe aux yeux des « résistés » (les contremaîtres par exemple) et, pourrait-on ajouter, aux yeux de tierces personnes. Le village de tentes installé dans le parc organise chaque mois un café-rencontre ouvert à tous qui s'installe pendant deux heures dans l'espace public, près de l'entrée du parc. Les organisateurs et les participants n'ont pas nécessairement l'impression de faire là acte de résistance, mais les comptes-rendus de l'assemblée de la mairie d'arrondissement font apparaître que l'un des élus 64 a évoqué l'affaire à au moins deux reprises 65 (mars et juin 2014) en demandant que cesse cette occupation de l'espace public, à ses yeux inacceptable. De même, si, pour les nojukusha, leur mode d'existence et notamment leurs installations et réinstallations dans des lieux publics ou semi-publics sont d'abord filles de la nécessité et ne prennent que ponctuellement la forme d'une résistance active, pour les services de la mairie chargés des parcs et cours d'eaux, c'est un obstacle dont la réapparition récurrente s'apparente à une forme de résistance aux projets menés. Pour un tiers, la présence et les activités des nojukusha dans un arrondissement où les projets de rénovation urbaine se multiplient et où la gentrification gagne du terrain peuvent aussi être perçues comme une force de résistance aux changements en cours.

\section{Résistance pour soi et résistance en soi}

Il y a ainsi à la fois des formes de résistance clairement intentionnelles qu'on pourrait appeler résistance pour soi pour reprendre en la modifiant l'opposition classique entre « classe en soi » et «classe pour soi ». La résistance pour soi est une résistance consciente d'elle-même. À l'inverse, on peut parler de résistance en soi pour désigner les formes de résistance dont l'intentionnalité n'est pas avérée mais qui sont perçues comme telles par les « résistés » ou par des tiers - la résistance en soi est en fait une résistance pour d'autres. Contrairement à l'opposition traditionnelle entre « classe en soi » et « classe pour soi ", qui fait de la première, pour ainsi dire, une forme inachevée de la seconde, il n'y a pas de hiérarchie a priori entre les deux formes de résistance. La perception des résistés et des tiers fait parfois apparaître la portée profondément subversive de pratiques qui, pour leurs acteurs, ne poursuivaient que des objectifs très terre-à-terre liés aux nécessités de l'existence. Les braconniers décrits par E. P. Thompson chassent pour se nourrir ou améliorer leur quotidien - il n'y a là, semble-t-il, aucune forme de résistance pour soi. Mais pour les juges qui, en application du Black Act (1723) les condamnent à mort, leurs actes sont une atteinte aux droits des propriétaires des forêts et à l'ordre

63. Prasad A., Prasad P., «(Un)willing to Resist? The Discursive Production of Local Workplace Opposition ", Studies in Cultures, Organizations and Societies, vol. 7, n 1, 2001, pp. 121-122.

64. Il ne s'agit pas de l'élu ayant initié le reportage sur le recyclage des canettes.

65. Le journal des débats à l'assemblée est publié sur le site de la mairie d'arrondissement. Pour conserver l'anonymat des personnes concernées, je n'indique pas non plus ici le nom de l'arrondissement. 
établi, en même temps qu'une remise en question de l'état actuel des rapports de force entre classes sociales - résistance en soi et résistance pour d'autres. Le mode de vie et les activités des nojukusha, leurs pratiques de contournement et de grignotage ne peuvent pas toujours être facilement saisies comme une résistance en soi, mais elles constituent pour l'élu ayant incité à la réalisation du téléreportage évoqué une série de comportements déviants qui résistent à la mise en ordre de l'espace socio-urbain. De même, la construction d'espaces autonomes, comme ceux institués par les pratiques agricoles décrites, et l'émancipation, si elles ne sont pas nécessairement des formes de résistance pour soi, peuvent être perçues par les résistés ou des tiers comme des formes de résistance en soi, puisqu'elles remettent en cause l'ordre établi, c'est-à-dire le système des rapports sociaux de pouvoir.

En somme, la question de l'intentionnalité de la résistance est importante si on se place au niveau des individus et des groupes impliqués ; mais si l'on change d'échelle et replace la résistance dans le triangle qui la configure, c'est bien plus l'effet que l'intention qui importe - la résistance en soi, plus que la résistance pour soi. Cette distinction entre deux formes de résistance permet ainsi de replacer les pratiques de résistance dans la dynamique plus globale des rapports de pouvoir en cours. On aperçoit mieux alors, dans les six types de pratiques politiques subalternes présentées - renversement des rapports de pouvoir, négociation, contournement, grignotage, création d'espaces autonomies et pratiques émancipatrices - ce qui fait résistance et pour qui.

Renversement des rapports de pouvoir et négociation sont des formes claires de résistance pour soi, où la résistance intentionnelle des acteurs impliqués prend la forme d'une confrontation visant à défendre des acquis - le « droit d'usage » de la collecte des canettes - ou à s'opposer à des mesures les affectant - mobilisations contre des procédures d'expulsion... Cette résistance intentionnelle est en général aussi perçue comme telle par les résisté-e-s ou des tiers, et partant, elle est aussi résistance en soi. Les pratiques de contournement et grignotage, qui maintiennent un dialogue, tacite ou non, avec les dominants peuvent, selon les cas, mais de manière souvent moins claire, être considérées comme des formes de résistance pour soi: la construction d'une tente sous une autoroute aérienne ne paraît pas pouvoir être considérée clairement comme une forme de résistance pour soi, pour les raisons indiquées ; la reconstruction d'une tente dans un endroit d'où l'on a été délogé pourrait l'être un peu plus, mais là encore, il est possible que ce soient plus les services de la mairie que la personne concernée qui perçoivent cet acte comme une résistance - résistance en soi donc, plus clairement que résistance pour soi. Création d'espaces autonomes et pratiques émancipatrices ne sont, elles, clairement pas des formes de résistance pour soi, puisqu'au contraire, elles supposent dans les deux cas de chercher à sortir du rapport de pouvoir défavorable, c'est-à-dire aussi du cou- 
ple domination-résistance. De ce fait, ce sont elles qui, en cherchant à s'en extraire, remettent le plus fortement en question les rapports de pouvoir et qui, de par leur portée subversive, peuvent être perçues par les dominants ou des tiers comme des formes effectives de résistance en soi, alors même que l'horizon des acteurs se place déjà en fait au-delà des rapports de pouvoir opposant résistance et domination. 